1 Flying in the Face of Adversity: A Drosophila-based Virtual CURE Provides Semester-long Authentic

2 Research Opportunity to the Flipped Classroom.

3 Edward A. Waddell, $\mathrm{PhD}^{1,2}$; Dara Ruiz-Whalen ${ }^{3}$, Alana M. O'Reilly ${ }^{3}$ Nathan T. Fried, $\mathrm{PhD}^{4 *}$

$4 \quad{ }^{1}$ Department of Biology, Holy Family University, Philadelphia, PA, USA

$5 \quad{ }^{2}$ Department of Neuroscience, Perelman School of Medicine, University of Pennsylvania, Philadelphia, PA

$6 \quad{ }^{3}$ eCLOSE Institute, Philadelphia, PA, USA

$7 \quad{ }^{4}$ Department of Biology, Rutgers University, Camden, NJ, USA

$8{ }^{*}$ Corresponding author

9 Corresponding Author Contact Nathan Fried, PhD nathan.fried@rutgers.edu

15 Sources of Support: provided funds for collaboration between authors.

21 Running head or foot line of approximately 40 characters: Drosophila-based Virtual CURE

22 Number of Figures: 2

23 Number of Tables: 2

24 Number of Supplemental Materials: 11

25 Word Count: 3990 


\section{Conflict of Interest Notification:}

3 The corresponding author, Nathan T. Fried, and Edward A. Waddell declare no potential conflicts of interest with

4 respect to the research, authorship, and/or publication of this article.

5

6 Dara Ruiz-Whalen and Alana M. O'Reilly declare that they are the Chief Learning and Chief Scientific Officers,

7 respectively, for the Philadelphia-based non-profit organization, eCLOSE Institute. 
1 Abstract: A call for the integration of research experiences into all biology curricula has been a major goal for 2 educational reform efforts nationally. Course-Based Undergraduate Research Experiences (CUREs) have been the

3 predominant method of accomplishing this, but their associated costs and complex design can limit their wide

4 adoption. In 2020, the COVID-19 pandemic forced programs to identify unique ways to still provide authentic

5 research experiences while students were virtual. We report here a full guide for the successful implementation of a

6 semester-long virtual CURE that uses Drosophila behavioral assays to explore the connection between pain and

7 addiction with the use of a "lab-in-a-box" sent home to students. Individual components were piloted across three

8 semesters and launched as a 100-level introductory course with 19 students. We found that this course increased

9 science identity and successfully improved key research competencies as per the Undergraduate Research Student

10 Self-Assessment (URSSA) survey. This course is ideal for flipped classrooms ranging from introductory biology to

11 upper-level neuroscience courses and can be integrated directly into the lecture period without the need for building

12 a new course. Given the low cost, recent comfort with virtual learning environments, and the current proliferation of

13 flipped biology classrooms following the 2020 pandemic, this curriculum could serve as an ideal project-based

14 active-learning tool for equitably increasing access to authentic research experiences.

15

16 Keywords: CURE, virtual, active-learning, backwards design, pain, addiction, Drosophila, biology, science identity 


\section{Introduction:}

2 For students to develop critical thinking skills as learners, scientists, and citizens, they must participate in activities

3 that apply both practical research techniques and the scientific process in real-world contexts $(1,2)$. Undergraduates

4 can accomplish this through mentored research experiences which are a major goal of educational reform efforts.

5 These are integral to biology education as they provide a high academic challenge, active collaborative and

6 experiential learning, enriching educational experience, intense student-faculty interaction, and a supportive campus

7 environment (3). These activities, however, are not widely available to all students due to high student-faculty ratios

8 and challenges integrating authentic research experiences into the biology curricula. Given the growing comfort-

9 level with virtual learning environments post-COVID and the subsequent wide movement toward pre-recorded

10 lectures, a unique opportunity is presented to integrate a project-based authentic research experience directly into the

11 classroom's weekly three-hour lecture period in either an in-person or virtual setting. By strategically focusing on

12 behavioral assays as experimental outputs, costs can also be dramatically reduced.

14 Considerable research has demonstrated the benefits of undergraduate research experiences on student learning,

15 including the development of domain expertise, acquisition of team-based skills, increased understanding and

16 respect for the research process, acquisition of problem-solving skills, practice and refinement of communication

17 skills, and increased self-confidence, personal growth, independence, and tolerance (4-10). Comparable benefits are

18 seen across race and gender and across various institutional types including research universities, Master's-level

19 institutions, and teaching colleges (11). Furthermore, these experiences are hypothesized to especially benefit

20 women and underrepresented students due to the fostering of mentor-mentee and peer-peer relationships (12-14).

\section{1}

22 Despite these recommendations, however, the practicalities of expanding this to an entire undergraduate population

23 is extremely daunting $(15,16)$. Historically, the incorporation of research experiences into the undergraduate

24 curriculum has been in the form of mentored one-on-one research apprenticeships in faculty research laboratories

$25(5,11)$. However, the high student-faculty ratio often prevents all students from participating, often at the cost of

26 diversity and equity in training $(17,18)$. A solution to this problem is to integrate authentic research experiences into

27 a course-based setting. 
1 Course-based undergraduate research experiences (CUREs) are a scalable solution for providing authentic research

2 experiences to undergraduate students while still fulfilling the benchmarks set by the National Research Council (8,

3 19). CUREs offer the capacity to involve a greater number of students with diverse backgrounds in research for

4 credit towards their degrees, not just self-selected students who seek out individual research opportunities (20). This

5 is especially the case for students of low socioeconomic backgrounds who often are unable to participate in

6 extracurricular research activities due to employment. Additionally, CUREs can be integrated into introductory-level

7 courses to engage first- and second-year students in research much earlier and thus have the potential to exert a

8 larger influence on students' academic and career choices (21).

10 Despite the numerous implementations and successes of CUREs, they are still widely underused. Since CUREs are

11 generally offered in a teaching laboratory versus an established research laboratory, a dedicated laboratory space to

12 create a scalable, authentic research experience may not be feasible for some institutions. Further, CUREs are often

13 stand-alone electives which can disincentive programs adopting them because they can be perceived as too

14 cumbersome for packed curricula or too time-consuming for overworked faculty. One way to overcome this is to

15 create viable authentic research experiences that can be integrated directly into the lecture-period and/or allow

16 students to conduct research at home. As higher education begins to place a greater priority on quality online

17 education, virtual CUREs can serve as a means to increase undergraduate participation in authentic research

18 experiences and have already seen some success (22).

19

20 Here we report the implementation of a virtual CURE (vCURE) geared toward first-year undergraduates at Rutgers

21 University Camden, a primarily undergraduate institution with a diverse and nontraditional STEM student

22 population (90\% commuters, 55\% first-generation/low income, $28 \%$ African American, and 16\% LatinX). The

23 course allowed students to explore the intersection between the opioid epidemic and pain using the model organism

24 Drosophila melanogaster at a cost of less than $\$ 15 /$ student. Through self-report data, we find that this course

25 improved science identity and key research competencies as assessed by the Undergraduate Research Student Self-

26 Assessment (URSSA) survey $(23,24)$. This manuscript describes the structure and function of this 3-credit vCURE

27 model and provides detail to adopt it in its entirety or adapt it to fit specific needs. Uniquely, our vCURE could be

28 implemented in a hybrid manner as a project-based research experience that serves as the active-learning component 
1 of flipped classrooms ranging from Biology 101 to upper-level Neuroscience. We also describe details of tapping

2 into the proliferation of virtual scientific conferences/webinars as a method to provide students with a unique

3 training opportunity often missed by the financial constraints of attending in-person conferences. It's our hope that

4 this vCURE model could be widely and easily adopted for use nationally to increase access to authentic research

5 experiences at any stage of the undergraduate curricula.

6

7 Intended audience: The intended audience is first-year biology majors but is designed to run at any undergraduate

8 level. Additionally, we also had a handful of non-biology majors, suggesting it could be effectively run for a range

9 of undergraduates.

11 Learning time: This is a semester-long project-based 3-credit biology elective across 15 weeks. However, individual

12 components of the course can be adopted to fit into smaller time periods. Students can expect weekly to spend 1

13 hour watching pre-lecture videos, 3 hours in synchronous sessions, and 1-6 hours in asynchronous sessions working

14 on the project or assignments.

15

16 Prerequisite student knowledge: There is no prerequisite knowledge and students do not need experience working

17 with Drosophila or in a lab setting.

18

19 Learning objectives:

1. Understand molecular mechanisms of addiction. (Comprehension)

2. Describe molecular mechanisms by which pain is governed. (Comprehension)

3. Demonstrate how to perform a search for primary literature. (Application)

4. Use Drosophila melanogaster laboratory techniques to address research questions. (Application)

5. Apply laboratory techniques to test hypotheses. (Application)

6. Generate a hypothesis based on primary literature. (Synthesis)

7. Critique scientific talks from a national conference. (Synthesis)

8. Support hypothesis with data. (Evaluation) 


\section{Learning Outcomes:}

1. Describe neurological concepts of pain. (Comprehension)

2. Explain neurological concepts of addiction. (Comprehension) 


\section{Procedure:}

2 This course integrates experiential learning into a flipped-classroom environment by implementing Drosophila-

3 based research into lecture periods. It is designed as a 3-credit 15-week course that meets twice/week but can be

4 modified as the instructor sees fit to run in a shorter curriculum. While this course was run in and is ideal for a

5 virtual setting, the original concept was developed for an in-person environment to place a research-based project

6 into a traditional 3-credit flipped classroom without the need for including an additional co- requisite lab.

8 Each week is broken into two days: 1) content days where in-class discussions/activities focus on understanding

9 required content and 2) lab meetings where in-class discussions/activities focus on completing a semester-long

10 research project. Student assignments/activities are broken into "individual work" and "group work". All work and

11 activities are designed to mirror that which would be experienced in a traditional research lab.

12

13 Below are information/materials necessary to run this course within the scope of students learning about the

14 neuroscience of addiction and chronic pain. However, the components can be used for other topics, and other

15 protocols could be adopted to the content of interest. Thus, we have written this manuscript to facilitate faculty

16 implementing the curriculum in its entirety or identifying individual components to adopt/modify/supplement their

17 courses.

18

19 Materials: Students will need the following:

20 1. Computer/internet access.

212 2. Video conferencing platform such as Zoom for synchronous meetings.

22 3. Cloud-based word processor/database/presentation programs such as those provided by Google Docs for 23 collaborative work.

24 4. Communication platform for instant conversation/troubleshooting such as Slack.

25 5. Learning management software for classroom organization such as Canvas.

26 6. "Lab-in-a-box" that includes Drosophila and all necessary tools for students to carry out the behavioral experiments in the semester-long research project. This "lab-in-a-box" is described within the Lab Manual 
(Appendix 4) and Assembly Plan (Appendix 5).

2

3

\section{$\underline{\text { Student Instructions: }}$}

Students are responsible for both individual and group work in the form of leading synchronous/asynchronous discussions, quizzes, presentations, and hands-on research activities. Detailed student instructions for each component can be found in the Course Syllabus (Appendix 1), Course schedule (Appendix 2), Journal club worksheet (Appendix 3), Lab Manual (Appendix 4), and Peer assessments (Appendix 6).

\section{Faculty Instructions:}

Below are instructions, commentary, and advice for the successful implementation of each component of the course. Further commentary on each component can be found in the Syllabus Annotations (Appendix $1)$.

Designing the Research Project: Faculty should design a semester-long research project that is easily approachable by students in the format of a traditional CURE. We recommend using backwards design that starts with a straight-forward research question/hypothesis/prediction and includes simple experimental design/variables. These can be constructed, as done here, by using Drosophila and simple behavioral assays. Once these are established, all other course content can be developed. We advise balancing impact with simplicity when designing this. For our project, we utilized four simple behavioral assays to study the impact of chronic pain on the development of addiction in Drosophila.

2

Given the limited in-class time, project plans should be fully developed prior to starting the course. See the Lab Manual for details on our project (Appendix 4). Faculty may feel free to use our project design to explore the same question, a similar question, build a different project based off the included behavioral assays, or develop an entirely different research project. We encourage student feedback when designing these variables but within reason to ensure the project has scientific authenticity and is not simply repeating existing published findings. For example, while we explored the impact of chronic pain on addiction, 
students may be interested in exploring how sleep, diet, or other factors impact addiction. Exploring an unknown instead of confirmatory experimentation instills greater buy-in from students and is the driving essence of authentic CUREs. We also suggest using the simple-to-approach behavioral assays. We used the negative geotaxis, sensitivity, tolerance, and Capillary Feeder (CAFE) assays to assess addiction. If built around simple and consistent behavioral assays as the dependent variable, faculty can explore a range of relevant topics as independent variables.

Conducting the Research Project: We include details for building a "lab-in-a-box" at the cost of approximately $\$ 15 /$ student. If deviating from our project design, we recommend taking time to consider all necessary equipment, including gloves, paper towels, additional fly food, and vials. We also recommend waiting to send this "lab-in-a-box" until the experiments actually begin (approximately week 5) since flies need to be flipped to new vials regularly and students do not begin their experiments until week 5/6.

While students are broken into groups of 4-5, each student is responsible for collecting a single set of experimental data points for each assay (i.e., every student will conduct all experiments instead of a single group being assigned a single assay). Thus, a student should complete a set number of replicates, but each student's data will be considered a single " $\mathrm{n}$ " to ensure variability of environment is accounted for, especially in virtual settings. This helps validate student results if the data are to be published. It is to be expected that not all students will produce sufficient data as all experimentation features potential methodological failures or impassable troubleshooting. Students should be reassured that this is a natural part of research and should not feel pressure to rush any experiments to preserve reliability of the data. Additionally, some students may wish to abstain from certain experimental procedures due to personal ethics or discomfort with flies. These students can virtually conduct the experiments with peers to still gain the underlying concepts and knowledge of the experiment. 
their particular presentation. This may include groups creating a central repository with a cloud-based software for data upload.

Content Days: The first session of the week is a lecture period that includes active learning and traditional lectures that reviews essential content. Critically, the content should be identified with backward design framed around the goals of the overarching research project. For example, we identified critical knowledge necessary to understand the project (basic neuroscience, neuroanatomy of pain/addiction, Drosophila biology, etc.) and used backwards design to build the course content structure. These days include prelecture videos (Appendix 2), weekly quizzes, presentation of recent science news, and traditional lecture content.

Lab Meeting Days: The second session of the week includes group-based journal clubs, presentations, and technique instruction which can be seen in the Course Schedule (Appendix 2). The first few weeks reinforce concepts in experimental design, responsible conduct of research, basic statistical analysis, and presentation of data. The following weeks focus on group-led journal clubs related to the research project. Once students have received their "lab-in-a-box", faculty use this time to instruct students on experimental techniques. We recorded these sessions for future reference and used break-out rooms for troubleshooting. Once students begin to collect data, these sessions are then used for the group-led research presentations.

Suggestions for Determining Student Learning: Rubrics are included with all assignments. In addition to each rubric, two peer surveys are provided: 1) Peer Evaluation - group members rate each other's involvement. 2) Presentation Peer Feedback - non-group members rate presenting group. Weekly quizzes are designed not only to test comprehension of the pre-lecture videos, but also provide incentive to prepare for class discussions.

Sample data: We have provided examples of each of the four research presentations (proposal, RIP I, RIP II, and Thesis Defense), a Conference Debrief Presentation, a Journal Club Presentation, and a Journal Worksheet Example (Appendices 8-11). 
1 Safety issues: The procedures and contents were designed to comply with the American Society of Microbiology

2 Guidelines for Biosafety in Teaching Laboratories. Although none of the items in this "lab-in-a-box" are hazardous,

3 we recommend students attend an in-person or virtual lab safety training. We utilized an online CITI Right-To-

4 Know lab safety training program. Of note, however, these boxes contain live Drosophila, low concentration ethanol

5 (max 50\% EtOH in comparison to hand sanitizer at $60 \% \mathrm{EtOH}$ ), and small glass capillary tubes.

\section{Discussion:}

8 Field testing: This course was initially conceived as a strategy to integrate a low-cost CURE into a flipped classroom

9 as an in-person semester-long project-based activity. Whereas flipped classrooms often contain a series of active

10 learning exercises and CUREs are often their own separate entity, our curriculum can be plugged into a traditional

11 flipped lecture-based course. This allows for the expansion of CUREs while avoiding the common impediments to

12 introducing them more widely since any flipped course could rely on our CURE model for its active-learning

13 component. As such, we built this CURE around a popular course that ran in two previous semesters at the 400-level

14 called "The Neuroscience of the Opioid Epidemic".

15

16 We initially piloted the virtual aspect of this CURE in the summer of 2020 when we partnered with the non-profit

17 research hub "eCLOSE" that had developed a fully virtual bioscience research curriculum with a Drosophila-based

18 "lab-in-a-box" for students ranging from middle school to college. During that summer, four Rutgers Camden 19 undergraduates participated in this program along with 49 students from other institutions to pilot the "lab-in-a-box"

20 curriculum and identify methods to adapt it for use with our pain/addiction course plan. We combined this virtual

21 component with other mechanisms ideal for the lecture period that were developed and tested in our more traditional

22 300-level CURE; we then launched our lecture-period-based virtual CURE in the Fall of 2020. Thus, components of

23 this virtual CURE have been field-tested over several semesters, piloted in the summer of 2020, and fully tested in a

24 100-level undergraduate course with 19 honors students during the Fall of 2020 (See demographics for the 12 who

25 participated in the survey in Table 1).

27 Evidence of student learning: Learning objectives 1-2 and outcomes 1-2 were evaluated with the formative 28 assessments of weekly quizzes. Learning objectives 3 and 7 were assessed with journal club presentations, journal 
1 club worksheets and a final conference debrief presentation (Appendices 8, 10, 11). Notably, it was impressive to

2 see the students digest and discuss research articles in this field as primarily first-year students. We strongly believe

3 with guidance, non-honors students could also achieve similar outcomes. Learning objectives 5-6, 8 and outcomes

$4 \quad 3-5$ were assessed with the formative and summative assessments of research presentations (Appendix 9). For these

5 presentations, students must be able to conduct the experimental assays and collaborate with their peers outside their 6 group to consolidate, analyze, and present their data.

8 We additionally used two validated survey instruments in a pre-test/post-test design to assess the effectiveness of 9 our vCURE on several science-related outcomes. The pre-test took place at the end of the fourth week of class 10 before students received their "lab-in-a-box", but after they had a month to gain familiarity with the study and 11 content. This allowed us to examine the effects of the course's research component. 12 of the 19 students took part

12 in both the pre- and post-tests. We collected demographic data indicating first-generation college student status,

13 major, age, gender, and year (Table 1). To ensure these students were well matched, we also confirmed that they did

14 not have any previous research experience. However, we note their interest in science given their participation in a 15 range of extracurricular science-related activities (Table 1).

17 We first assessed science identity which is a measure of how much a student identifies as a scientist or science

18 trainee (23). This measure positively correlates with success, academic retention, and whether the student enters a

19 science occupation. On a 1-7 Likert scale, science identity significantly increased from $4.75 \pm 1.36$ to $5.33 \pm 0.89$ in

20 the full cohort (Figure 1A, $\mathrm{p}=0.0463$ ). We saw no differences when comparing first-generation to non-first-

21 generation students. Interestingly, science identity was significantly less in females than males at both the pre- and

22 post-timepoints (Figure 1B, C, p $<0.05$ and $<0.001$ respectively).

24 We also measured science identity discrepancy which assesses the difference between how a student perceives

25 themselves in relation to how they perceive others see them in science. This comparison creates a scale where more

26 positive numbers indicate a student rates themselves less than they think others would rate them in science. Thus,

27 positive values could be interpreted as a measure of "science imposter syndrome" while negative values could be

28 interpreted as a measure of "science underdog status". While no changes were seen in the full cohort comparing pre- 
1 to post-, we did see gender differences at each time point (Figure 1D). At both the pre- and post- timepoints, males

2 had neutral science discrepancies (Figure 1E). Females, however, had greater discrepancy scores than males at both

3 time points, suggesting greater levels of imposter syndrome (Figure 1F). There were no differences between first-

4 generation and non-first-generation students.

6 We then used the URSSA survey to evaluate whether our vCURE was a successful program (24). This validated 34-

7 question survey reports four critical research measures: Thinking and Working Like a Scientist, Personal Scientific

8 Gains, Scientific Skills, and Researcher Attitudes/Behaviors. We found significant increases in all four measures

9 across the total cohort (Figure 2) but no differences between first-gen and non-first-gen students or between genders.

10 Notably, we find no differences amongst any of the groups in regard to their baseline research confidence which is a

11 measure of self-efficacy to perform science-related tasks (Table 2). Combined with the gender differences in science

12 identity discrepancy, we posit that while female students within our class may feel less capable than those around

13 them, a quantified self-assessment of their own ability suggests otherwise.

15 These gender differences are not necessarily surprising since females in several fields indicate greater imposter 16 syndrome than males $(25,26)$. However, it reinforces the importance of still pursuing systemic improvements that

17 encourage women in STEM regardless that the National Institutes of Health no longer considers women as

18 underrepresented in the biomedical sciences. While we didn't see any differences in first-generation students, the

19 URSSA assessments may instead suggest that this vCURE can be effective regardless of familial college experience.

21 Possible modifications: With the inevitable proliferation of flipped classrooms after COVID-19 forced most biology

22 faculty to pre-record their lectures for the first time, we believe there is an unprecedented opportunity for integrating

23 CUREs directly into the lecture-period as the active learning component of a flipped classroom. Given that our

24 CURE could be run in either a virtual or in-person capacity, we are confident this model could be widely adopted.

25 Further, the recent comfort with virtual settings could expand the accessibility of research experiences by adopting

26 this vCURE into a range of settings such summer bridges or REUs (Research Experiences for Undergraduates)

27 without the need for expansive infrastructure or large budgets. 
1 While we ran this as a full 3-credit 15-week course, it could be consolidated to a shorter period by removing

2 experiments. Combined with its low-cost and virtual capacity, it could be integrated into non-curricular activities

3 such as summer bridge programs. This vCURE could be a low-cost method to foster science identity prior to a

4 student entering their first college-level biology course. It could also easily be scaled up to large entry-level biology

5 courses which have traditionally found it challenging to integrate active learning. Further, these activities are

6 beneficial for students at any level and thus could be used in upper-level courses.

8 Our model can be modified to function in either a virtual or in-person setting and could be connected to any

9 scaffolded biology course. The course could also be adopted in its entirety or specific components integrated into

10 other curricula. Further, the behavioral assays could be adopted for research studies exploring a wide range of

11 research topics outside the realm of pain and addiction.

12

13 One of the defining characteristics of immersing oneself fully into the scientific community is interacting with peers

14 and colleagues at scientific conferences. This essential experience is often restrictive to undergraduate students

15 either due to financial reasons constraints of travel or registering. As conferences begin to move back to in-person

16 settings, we are confident webinars and virtual seminars will continue to be accessible for students to tap into within

17 the structure of our vCURE.

18

19 Acknowledgements: We thank the many Rutgers Camden undergraduates who provided their feedback as we

20 developed and launched this course. We thank Dr. Kwangwon Lee who provided mentorship and guidance to make

21 this course and manuscript a success. We also thank the Rutgers Camden Honors Program and Provost Office for

22 funding the "lab-in-a-box" and the NIH K12 IRACDA PennPORT program (K12-GM081259) for funding the

23 virtual conference travel. Finally, we thank the American Society for Cell Biology (ASCB) Promoting Active

24 Learning and Mentoring (PALM) Network for their funding that allowed the authors to develop this course.

25

\section{References:}

27 1. Laursen S, Hunter A-B, Seymour E, Thiry H, Melton G. 2010. Undergraduate Research in the Sciences:

28 Engaging Students in Real Science1st edition. Jossey-Bass, San Francisco. 
1 2. Felten P. 2013. Principles of good practice in SoTL. 1. Teaching \& Learning Inquiry 1:121-125.

2 3. Kuh GD. 2003. What We're Learning About Student Engagement From NSSE: Benchmarks for Effective 3 Educational Practices. Change: The Magazine of Higher Learning 35:24-32.

4 4. Katkin W. 2003. The Boyer Commission Report and its Impact on Undergraduate Research. New Directions 5 for Teaching and Learning 2003:19-38.

6 5. Lopatto D. 2004. Survey of Undergraduate Research Experiences (SURE): First Findings. Cell Biol Educ $7 \quad 3: 270-277$.

8 6. Gates AQ, Teller PJ, Bernat A, Delgado N, Della-Piana CK. 1998. Meeting the challenge of expanding 9 participation in the undergraduate research experience, p. 1133-1138. In Proceedings of the 28th Annual 10 Frontiers in Education - Volume 03. IEEE Computer Society, USA.

11 7. Hathaway R, Nagda B, Gregerman S. 2002. The relationship of undergraduate research participation to 12 graduate and professional education pursuit: An empirical study. J Coll Stud Dev 43:614-631.

13 8. Graham MJ, Frederick J, Byars-Winston A, Hunter A-B, Handelsman J. 2013. Increasing Persistence of $14 \quad$ College Students in STEM. Science 341:1455-1456.

159 9. Russell CB, Weaver GC. 2011. A comparative study of traditional, inquiry-based, and research-based 16 laboratory curricula: impacts on understanding of the nature of science. Chem Educ Res Pract 12:57-67.

17 10. Seymour E, Hunter A-B, Laursen SL, DeAntoni T. 2004. Establishing the benefits of research experiences for 18 undergraduates in the sciences: First findings from a three-year study. Science Education 88:493-534.

19 11. Lopatto D. 2007. Undergraduate Research Experiences Support Science Career Decisions and Active 20 Learning. CBE Life Sci Educ 6:297-306.

21 12. Nagda BA, Gregerman SR, Jonides J, von Hippel W, Lerner JS. 1998. Undergraduate Student-Faculty 22 Research Partnerships Affect Studen Retention. The Review of Higher Education 22:55-72. 
1 13. Estrada M, Woodcock A, Hernandez PR, Schultz PW. 2011. Toward a Model of Social Influence that Explains Minority Student Integration into the Scientific Community. J Educ Psychol 103:206-222.

14. Corwin LA, Runyon CR, Ghanem E, Sandy M, Clark G, Palmer GC, Reichler S, Rodenbusch SE, Dolan EL. 2018. Effects of Discovery, Iteration, and Collaboration in Laboratory Courses on Undergraduates' Research Career Intentions Fully Mediated by Student Ownership. LSE 17:ar20.

15. Olson S, Riordan DG. 2012. Engage to Excel: Producing One Million Additional College Graduates with Degrees in Science, Technology, Engineering, and Mathematics. Report to the President. Executive Office of

16. Corwin LA, Runyon C, Robinson A, Dolan EL. 2015. The Laboratory Course Assessment Survey: A Tool to Measure Three Dimensions of Research-Course Design. CBE Life Sci Educ 14:ar37. Research More Inclusive. LSE 13:602-606.

18. Linn MC, Palmer E, Baranger A, Gerard E, Stone E. 2015. Undergraduate research experiences: Impacts and opportunities. Science 347.

19. Rodenbusch SE, Hernandez PR, Simmons SL, Dolan EL. 2016. Early Engagement in Course-Based Research Increases Graduation Rates and Completion of Science, Engineering, and Mathematics Degrees. CBE Life Sci Educ 15.

20. Rowland SL, Lawrie GA, Behrendorff JBYH, Gillam EMJ. 2012. Is the undergraduate research experience (URE) always best?: The power of choice in a bifurcated practical stream for a large introductory biochemistry class. Biochemistry and Molecular Biology Education 40:46-62.

21 21. Hunter A-B, Laursen SL, Seymour E. 2007. Becoming a scientist: The role of undergraduate research in students' cognitive, personal, and professional development. Science Education 91:36-74. 
1 22. Sun E, Graves ML, Oliver DC. 2020. Propelling a Course-Based Undergraduate Research Experience Using an Open-Access Online Undergraduate Research Journal. Front Microbiol 11.

3 23. Stets JE, Brenner PS, Burke PJ, Serpe RT. 2017. The science identity and entering a science occupation. Social Science Research 64:1-14.

24. Weston TJ, Laursen SL. 2015. The Undergraduate Research Student Self-Assessment (URSSA): Validation for Use in Program Evaluation. CBE Life Sci Educ 14.

25. Feenstra S, Begeny CT, Ryan MK, Rink FA, Stoker JI, Jordan J. 2020. Contextualizing the Impostor “Syndrome.” Front Psychol 11.

26. Gibson-Beverly G, Schwartz JP. 2008. Attachment, Entitlement, and the Impostor Phenomenon in Female Graduate Students. Journal of College Counseling 11:119-132.

\section{Legends}

13 Figure 1: Science Identity Measures. Science Identity of full cohort at pre- and post-time points (A), male vs female

14 at pre- timepoint (B), and male vs female at post- timepoint (C). Science Identity Discrepancy of full cohort (D),

15 male vs female at pre- timepoint (E), and male vs female at post- timepoint (F). Pre- timepoint assessments occurred during week 4 of the course prior to receiving the "lab-in-a-box" while post- timepoint assessments occurred during

17 week 15 at the end of the experimentation period. $\mathrm{n}=12$ for full cohort ( 7 females, 5 males) with $*$ indicating $\mathrm{p}<$ 180.05.

20 Figure 2: URSSA Scores. Scores from the Undergraduate Research Student Self-Assessment for the full cohort:

21 Thinking and Working Like a Scientist (A), Personal Scientific Gains (B), Scientific Skills (C), and Researcher

22 Attitudes/Behaviors (D). Pre- timepoint assessments occurred during week 4 of the course prior to receiving the

23 "lab-in-a-box" while post- timepoint assessments occurred during week 15 at the end of the experimentation period.

$24 \mathrm{n}=12$ for full cohort with $*$ indicating $\mathrm{p}<0.05$. 
1 Table 1: Student demographics. SES refers to Socioeconomic Status. Science Experience is the percentage of

2 students involved in those associated activities related to science.

4 Table 2: Means \pm standard deviation for all survey instrument measurements and $\mathrm{p}$ values for all comparisons.

5 STETS refers to the Science Identity questionnaire developed by Stets et al. in 2017 that measures Science Identity

6 (SI), Science Identity Prominence (SP), and Science Identity Discrepancy (SD). RC indicates the Research

7 Confidence Questionnaire. URSSA is the Undergraduate Research Student Self-Assessment Measures developed by

8 Weston et al. in 2015 that measures thinking and working like a scientist, personal scientific gains, improvement of

9 scientific skills, and improvement of researcher attitudes/behaviors.

\section{Supplemental Materials:}

13 Student Documents:

Appendix 1: Course Syllabus - This course syllabus includes annotations for faculty within the comments to understand how to implement each portion of the course. 15-week course. Annotations for faculty within the comments are included for further guidance. Appendix 3: Journal Club Worksheet - This worksheet can be converted into an LMS quiz for faster grading and easier submission.

Appendix 4: Lab Manual - This lab manual includes the framing of the semester-long project, details on the "lab-in-a-box", and all protocols necessary to carry out the experiments.

\section{Faculty Documents:}

Appendix 5: "Lab-in-a-box" Assembly Plan - This document includes the assembly instructions, items, and details for where and how to purchase the items. 

presentations.

$4 \quad$ Example Student Work:

Appendix 8: Journal Club Worksheet Example - this is an example of a student's journal club worksheet. I/II, and Thesis Defense Presentations. group. conference debrief assignment.

12

13 Other: 
Figure 1
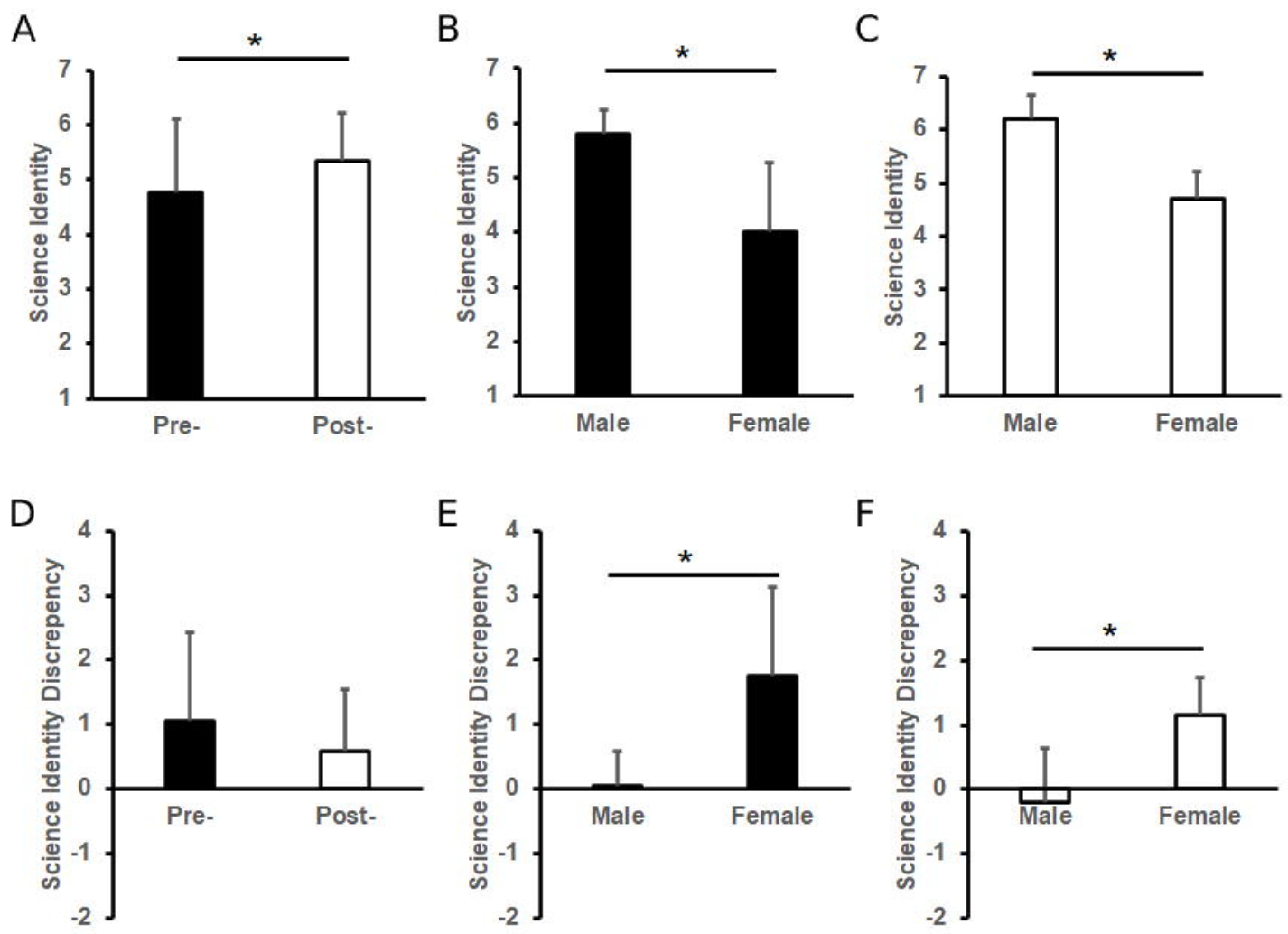
Figure 2

A

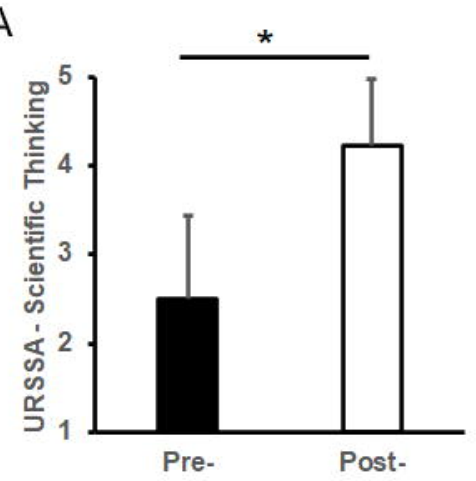

C

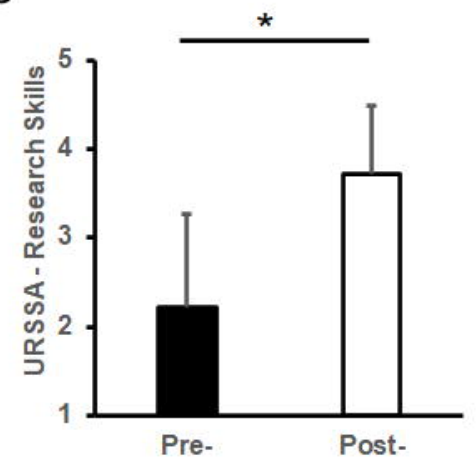

B

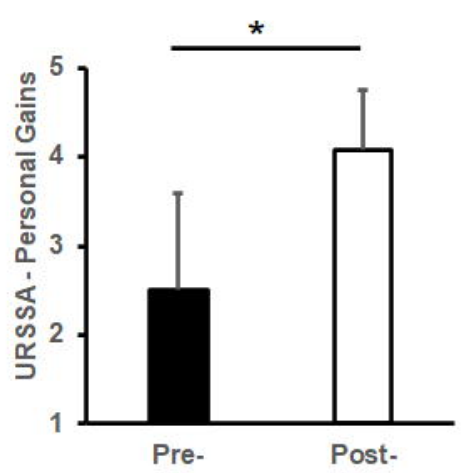

D

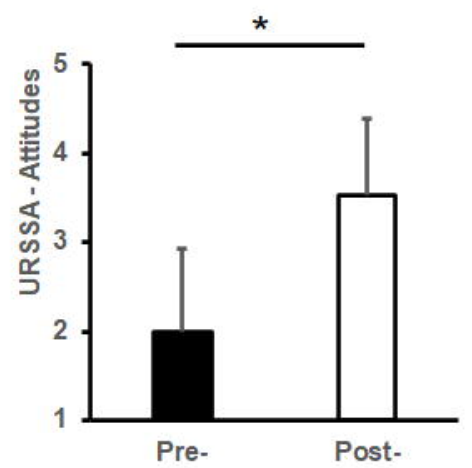




$$
\begin{aligned}
& \frac{5}{5}
\end{aligned}
$$

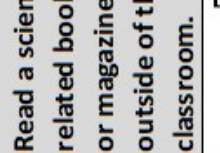

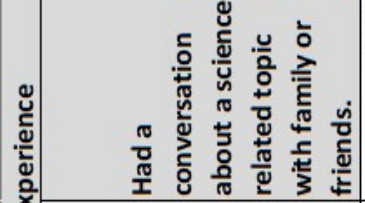

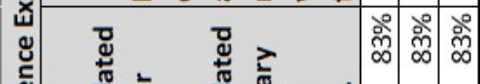
, 0
0

$8 \%$ II

.

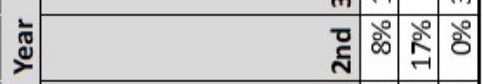

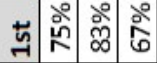

ธิ์

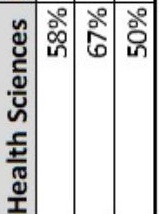

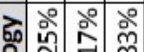

의 $\Rightarrow$

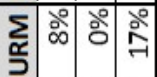

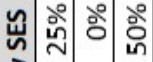

3

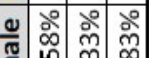

ह $\mathrm{m} \infty$ .

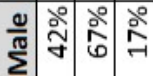
$\div \operatorname{mon}$ ᄃ

.

김

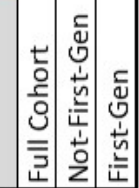

ㅎํㄴ 


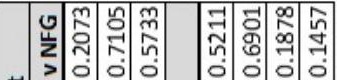

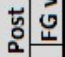

ப

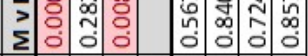

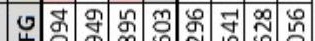
뜬 >

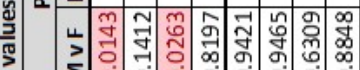
m
.

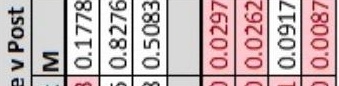
닌 웝

of 둥요

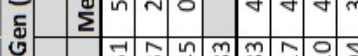

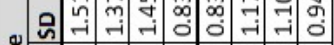

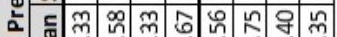

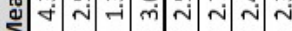

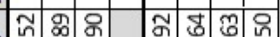

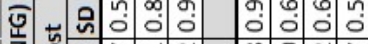

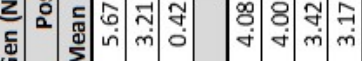

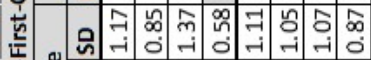
政

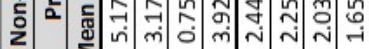

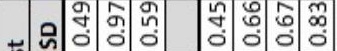

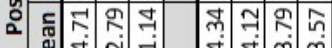
ह 글 0

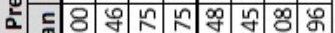

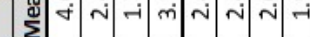

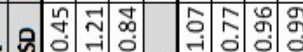
-10:

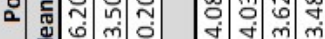

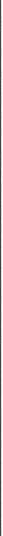
고공 *ssyn 